\title{
A Very Big Hand Is a Very Big Problem: Soft-Tissue Infection, Venous Thrombosis, or Just an Insect Sting?
}

\author{
Sarah Damanti ${ }^{1}$, Barbara Brignolo-Ottolini ${ }^{1}$, Marta Mansi ${ }^{1}$, Maura Marcucci ${ }^{2,3}$, Daniela Mari ${ }^{2,3}$, Valerio Pravettoni $^{4}$ \\ ${ }^{1}$ School of Specialization in Geriatrics and Gerontology, Università degli Studi di Milano, Milan, Italy \\ ${ }^{2}$ Geriatric Unit, Fondazione IRCCS Ca' Granda Ospedale Maggiore Policlinico, Milan, Italy \\ ${ }^{3}$ Department of Clinical Sciences \& Community Health, University of Milan, Milan, Italy \\ ${ }^{4}$ Allergology and Immunology Unit, Fondazione IRCCS Ca' Granda, Osp. Maggiore Policlinico, Milan, Italy
}

\section{Doi: 10.12890/2016_000312- European Journal of Case Reports in Internal Medicine - @ EFIM 2016}

\begin{abstract}
Received: 14/10/2015
Accepted: $16 / 11 / 2015$

Published: 20/01/2016
\end{abstract}

\begin{abstract}
How to cite this article: Damanti S, Brignolo-Ottolini B,Mansi M, Marcucci M, Mari M, Pravettoni P. A very big hand is a very big problem: soft-tissue infection, venous thrombosis, or just an insect sting? EJCRIM 2016;3:doi:10.12890/2016_000312
\end{abstract}

Conflicts of Interests: The authors declare that there are no competing interests.

This article is licensed under a Commons Attribution Non-Commercial 4.0 License

\section{ABSTRACT}

A 67-year-old woman developed severe edema of her right hand and forearm, for which she was treated with antibiotics, without benefit. The echography excluded a venous thrombosis. Subsequently, she referred a wasp sting before the development of the edema. Specific Hymenoptera venom immunoglobulin E (IgE) was found to be positive for paper wasp and yellow jacket. A large local reaction (LLR) was diagnosed due to the hymenoptera sting. Self-injectable epinephrine was prescribed for possible, though unlikely, systemic reactions following hymenoptera stings.

\section{LEARNING POINTS}

- The differential diagnosis of an upper forearm and hand oedema may be challenging.

- In order to reach the correct diagnosis and to prescribe proper treatment, careful examination and history taking is essential.

- All possible causes should be taken into proper consideration.

- Large local reaction (LLR) is characterized by hot, flushed and thick edema after a hymenoptera sting. As the risk of a systemic reaction, upon the first sting following a consistent index LLR, it is important to recognize an LLR in order to prescribe self-injectable epinephrine to prevent the occurrence of anaphylaxis.

\section{KEYWORDS}

Large local reaction, angioedema, anaphylaxis

\section{CASE REPORT}

A 67-year-old woman, while spending her holidays in the countryside, developed a severe edema of her right hand, which in 6 hours extended to the right forearm. The patient's clinical history disclosed hypertension, treated with an angiotensin converting enzyme (ACE) inhibitor, and allergic seasonal rhino-conjunctivitis from childhood and for which the patient had undergone subcutaneous specific immunotherapy for grass pollen. In the year 2014, when the patient was already taking the ACE inhibitor, she developed a lip and cheek angioedema after consuming hazelnut ice-cream. The symptoms remitted promptly following the administration of an antihistaminic drug. 
At physical examination, her vital signs were normal and peripheral arterial pulses were present. No lymphadenopathy was appreciated. The skin was hot and flushed, and there was a thick edema on the back of her hand and on her entire forearm (Fig. 1).

The doctor excluded erysipelas because of the lack of a typical raised edge and of a marked redness, but suspected a soft-tissue infection, for which he prescribed ciprofloxacin $500 \mathrm{mg}$, twice daily for 6 days. After 3 days of antibiotic treatment, the non-itching edema worsened and the patient developed pain and progressive functional impairment of hand and arm, as well. A venous ultrasound examination, promptly executed, ruled out venous thrombosis. At that time, the patient remembered she had been stung by a wasp on the back of her right hand the day prior to onset of the edema. She had been stung by wasps previously; the previous time was two weeks earlier, without any local reaction.

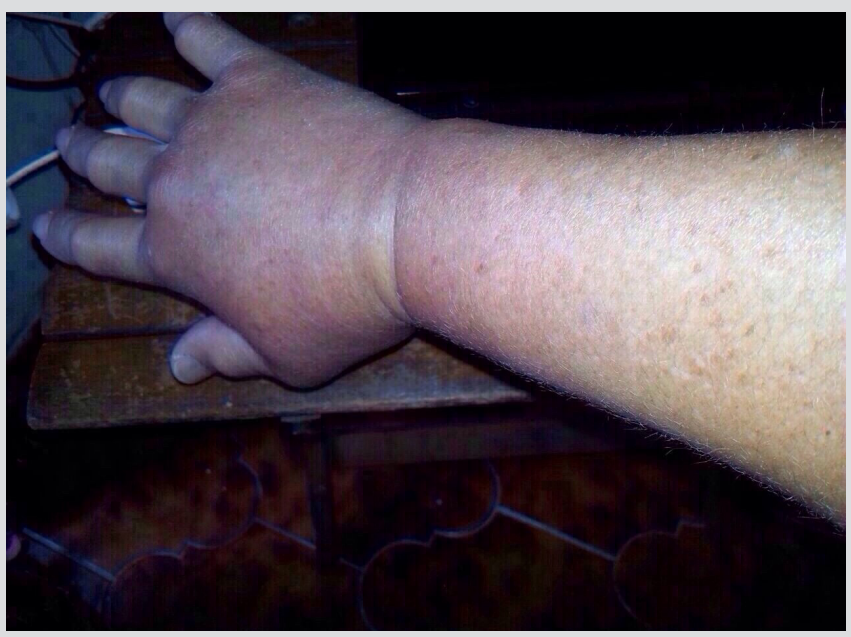

Figure 1: Flushed and thick edema of the patient's right hand and forearm

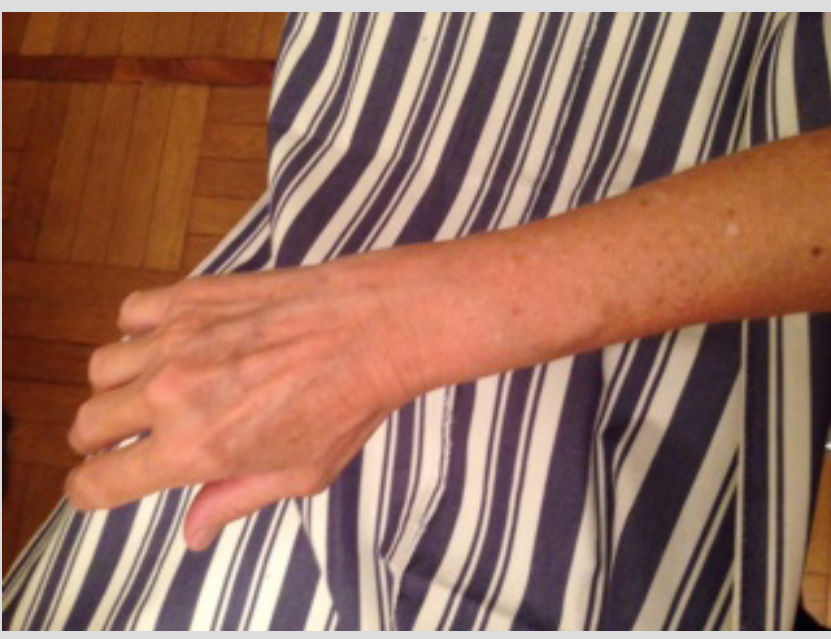

Figure 2: Resolution of the edema after the proper therapy

The patient was referred to an allergist; serum total immunoglobulin E (IgE), specific IgE to Hymenoptera venom, pollen and food allergens, serum tryptase, lactic dehydrogenase (LDH) and complete blood count (CBC) were measured. Local ice packs, oral prednisone and an antihistaminic were highly and rapidly effective in resolving the edema (Fig. 2).

CBC, serum tryptase and LDH were normal, excluding hematologic conditions such as mastocytosis and chronic lymphatic leukemia.

Total IgE were within the normal range; specific Hymenoptera venoms IgE were positive for paper wasp and yellow jacket. Specific IgE resulted positive for grass, birch, wall pellitory, and mugwarth pollens. In particular, IgE to recombinant birch allergens were positive only to the main birch pollen allergen: Bet v 1. Specific IgE to food were positive to hazelnut and peach, while negative to seed storage proteins and lipid transfer proteins. Interestingly, the raw peanut was negative, while the recombinant allergen, Ara h 8 , was positive.

The allergist interpreted the edema as a large local reaction (LLR), which had occurred after the hymenoptera sting and prescribed selfinjectable epinephrine to prevent a possible, though unlikely, systemic reaction following hymenoptera stings.

\section{DISCUSSION}

Hymenoptera LLR is a relatively rare (2.4-10\% ${ }^{[1]}$ occurrence, characterized by a large (mean diameter $\geq 10 \mathrm{~cm}$ ) area of edema and erythema appearing within a few minutes to several hours after hymenoptera stings, which worsen during the subsequent 24-48 hours. LLR may become indurated and painful and eventually interfere with the functional use of the affected area. It generally lasts for several days and may also be complicated by a non-infectious lymphangitis or by mild systemic symptoms like fever ${ }^{[2]}$. LLR may be caused by a toxic reaction, due to irritant components of the venom capable to produce direct damage to the skin and subcutaneous tissue.

Frequently, it occurs in patients sensitized to insect venom, and, in these circumstances, the onset is sudden. 
In our case, previous wasp stings, although devoid of symptoms, presumably caused the sensitization of the patient (positivity of specific Hymenoptera venoms IgE for paper wasp and yellow jacket), thus accounting for the rapid onset of the extended angioedema which, in this case, should be interpreted as a low-grade allergic reaction.

Our patient had an allergic history. The positivity of specific IgE homologous to Bet $v 1$ and of IgE to hazelnut indicates that the angioedema previously experienced after the assumption of a hazelnut ice-cream was a manifestation of the oral allergy syndrome related to pollen-food allergy. Allergens which cause this syndrome are labile to heat and pepsin digestion, thus restraining the symptoms only to the lips and the oral cavity. However, an allergic history does not constitute a risk factor for developing LLRs.

According to the international hymenoptera venom allergy guidelines ${ }^{[3]}$, as the risk of systemic reaction is relatively low (from 5 to $15 \%$ of LLR $)^{[1]}$, venom immunotherapy (VIT), though highly effective in venom allergic patients and conferring an almost complete protection against subsequent stings ${ }^{[3]}$, is not enforced.

Recent communications to the EAACI meeting ${ }^{[4]}$ suggest that the risk of systemic reactions after LLR is significant for the first sting after the index LLR. However, if the patient experiences an LLR again, all subsequent stings will never cause systemic reaction, being, in all likelihood, attended only by LLRs.

On this ground, as our patient had exhibited only one LLR, the allergist advised self-injectable epinephrine, in addition to the traditional treatment based on local ice dressing together with oral steroidal and antihistaminic drugs.

\section{REFERENCES}

1. Severino M, Bonadonna P, Passalacqua G. Large local reactions from stinging insects: from epidemiology to management. Curr Opin Allergy Clin Immunol 2009;9:334-337

2. Przybilla B, Ruëff F. Insect Stings Clinical Features and Management. Dtsch Arztebl Int 2012;109: 238-48

3. Bonifazi F, Jutel M, Bilo BM, et al. The EAACI Interest Group on Insect Venom Hypersensitivity. Prevention and treatment of Hymenoptera venom allergy: guidelines for clinical practice. Allergy 2005;60:1459-1470.

4. Pucci S, D'Alò S, De Pasquale T, Illuminati I, Makri E, Incorvaia C. Risk of anaphylaxis in patients with large local reactions to hymenoptera stings: a retrospective and prospective study. Clin Mol Allergy 2015;13:21 